\title{
Search-and-replace editing of genetic information
}

\author{
Yao LIU ${ }^{1}$, Xingxu HUANG $(\bowtie)^{2}$, Xiaolong WANG $(\bowtie)^{1}$ \\ 1 College of Animal Science and Technology, Northwest A\&F University, Yangling 712100, China \\ 2 School of Life Science and Technology, ShanghaiTech University, Shanghai 201210, China
}

Genome editing based on CRISPR/Cas9 system enables unprecedented breakthroughs in genomic research and the engineering of variants associated to clinical diseases ${ }^{[1,2]}$. The CRISPR/Cas 9 system induces double strand breaks (DSBs), which are primarily repaired by non-homologous end joining (NHEJ) and low-efficiency homologydirect repair (HDR) pathways. However, NHEJ can only randomly produce gene knockouts and HDR inefficiently induces sequence replacement ${ }^{[3]}$. To precisely edit a genome, the CRISPR-based cytosine base editor (CBE) and adenine base editor (ABE) were developed, facilitating C-to-T and A-to-G base substitutions, respectively, without requiring $D S B s^{[4,5]}$. Although many refinements have optimized $C B E$ and $A B E^{[6-10]}$, these editors can only induce base transitions. Also, they cannot achieve the editing of $C$ or $A$ at arbitrary positions due to limited editing windows and protospacer-adjacent motifs (PAMs). Moreover, unwanted bystander mutations can often be found within or near the protospacers, which impedes both the efficiency and fidelity of base editing ${ }^{[6]}$.

Recently, a search-and-replace genome editor, the prime editor (PE), was added to the genome editing toolkit ${ }^{[11]}$. PE combines an exogenous CRISPR/Cas9 system and endogenous DNA repair pathways and achieves superior editing versatility, inducing various types of base substitutions, small indels and combinations thereof. PE generally consists of three components, a Cas9 nickase-reverse transcriptase (RTase) fusion, an engineered prime editing guide RNA (pegRNA) and a single-guide RNA (sgRNA) ${ }^{[11]}$. By fusing Cas9 H840A nickase with Moloney murine leukemia virus RTase (M-MLV RTase), a versatile protein complex was constructed, which targets genome sites, nicks DNA, and initiates reverse transcription. The pegRNA encompasses a spacer region at the $5^{\prime}$ end, identically to a typical sgRNA. Moreover, it includes a primer binding site and a RT template at the 3' end, which triggers reverse transcription and harbors new genetic information respectively. This complex combining with pegRNA searches for and nicks the target DNA, and then, new genetic information is encoded into genome by reverse transcription. Meanwhile, an additional sgRNA nicks the non-edited strand and triggers the endogenous mismatch repair pathway. Then, the non-edited strand will be repaired using the edited strand as template ${ }^{[11]}$. Through these steps, precise genome editing can be achieved in a highly-directed manner.

Compared with previous CRISPR-based editing tools, PE can edit the regions near or distant from PAMs, thus overcoming the limitations of PAMs, and extending the scope of CRISPR-based genome editing ${ }^{[11]}$. However, similar to other tools that have been derived from the CRISPR system, PE can cause off-target effects; although, at a low frequency due to the unique applied editing strategies ${ }^{[11]}$. Furthermore, two nicks on target opposite DNA strands may cause more indels ${ }^{[12]}$. Sometimes, nicking sgRNAs with spacers that match the edited strand enables nicking of the non-editing strand after the first nick; this can be used to minimize indels ${ }^{[11]}$. In summary, PE has distinct advantages for genome editing but it is still at an early stage of development and additional work is required to optimize its targeting efficiency and to minimize any off-target effects.

As soon as the advantages of PE have been demonstrated in animals and plants, genomic research linking genotype to phenotype will benefit strongly ${ }^{[11,13]}$. With PE, most natural genetic mutations, that have been associated with biological function or diseases, can be engineered in cells or animals. PE will also be applicable to

Received January 6, 2020

Correspondences: huangxx@shanghaitech.edu.cn, xiaolongwang@nwafu.edu.cn 
several fields including agriculture and biomedicine. More complex genome editing can be conducted in livestock and crops, which will enable the rapid generation of novel mutants with economically important traits ${ }^{[14-16]}$. Moreover, the precision of PE indicates its low-security risk and promising prospects for gene therapy. In summary, $\mathrm{PE}$ is a more powerful CRISPR-based tool than base editors, can achieve almost any type of genome editing, and will profoundly influence biological studies and clinical applications.

\section{References}

1. Cong L, Ran F A, Cox D, Lin S, Barretto R, Habib N, Hsu P D, Wu X, Jiang W, Marraffini L A, Zhang F. Multiplex genome engineering using CRISPR/Cas systems. Science, 2013, 339(6121): 819-823

2. Komor A C, Badran A H, Liu D R. CRISPR-based technologies for the manipulation of eukaryotic genomes. Cell, 2017, 168(1-2): 20-36

3. Ceccaldi R, Rondinelli B, D’Andrea A D. Repair pathway choices and consequences at the double-strand break. Trends in Cell Biology, 2016,26 (1): $52-64$

4. Komor A C, Kim Y B, Packer M S, Zuris J A, Liu D R. Programmable editing of a target base in genomic DNA without double-stranded DNA cleavage. Nature, 2016, 533(7603): 420-424

5. Gaudelli N M, Komor A C, Rees H A, Packer M S, Badran A H, Bryson D I, Liu D R. Programmable base editing of A·T to G·C in genomic DNA without DNA cleavage. Nature, 2017, 551(7681): 464-471

6. Gehrke J M, Cervantes O, Clement M K, Wu Y, Zeng J, Bauer D E, Pinello L, Joung J K. An APOBEC3A-Cas9 base editor with minimized bystander and off-target activities. Nature Biotechnology, 2018, 36(10): 977-982

7. Kim Y B, Komor A C, Levy J M, Packer M S, Zhao K T, Liu D R. Increasing the genome-targeting scope and precision of base editing with engineered Cas9-cytidine deaminase fusions. Nature Biotechnology, 2017, 35(4): 371-376

8. Koblan L W, Doman J L, Wilson C, Levy J M, Tay T, Newby G A, Maianti J P, Raguram A, Liu D R. Improving cytidine and adenine base editors by expression optimization and ancestral reconstruction. Nature Biotechnology, 2018, 36(9): 843-846

9. Komor A C, Zhao K T, Packer M S, Gaudelli N M, Waterbury A L, Koblan L W, Kim Y B, Badran A H, Liu D R. Improved base excision repair inhibition and bacteriophage Mu Gam protein yields C:G-to-T:A base editors with higher efficiency and product purity. Science Advances, 2017, 3(8): eaao4774

10. Li J, Liu Z, Huang S, Wang X, Li G, Xu Y, Yu W, Chen S, Zhang Y, Ma H, Ke Z, Chen J, Sun Q, Huang X. Efficient base editing in G/C-rich regions to model androgen insensitivity syndrome. Cell Research, 2019, 29(2): 174-176

11. Anzalone A V, Randolph P B, Davis J R, Sousa A A, Koblan L W, Levy J M, Chen P J, Wilson C, Newby G A, Raguram A, Liu D R. Search-andreplace genome editing without double-strand breaks or donor DNA. Nature, 2019, 576(7785): 149-157

12. Shen B, Zhang W, Zhang J, Zhou J, Wang J, Chen L, Wang L, Hodgkins A, Iyer V, Huang X, Skarnes W C. Efficient genome modification by CRISPR-Cas9 nickase with minimal off-target effects. Nature Methods, 2014, 11(4): 399-402

13. Young A I, Benonisdottir S, Przeworski M, Kong A. Deconstructing the sources of genotype-phenotype associations in humans. Science, 2019, 365(6460): 1396-1400

14. Jenko J, Gorjanc G, Cleveland M A, Varshney R K, Whitelaw C B, Woolliams J A, Hickey J M. Potential of promotion of alleles by genome editing to improve quantitative traits in livestock breeding programs. Genetics, Selection, Evolution., 2015, 47(1): 55

15. Li G, Zhou S, Li C, Cai B, Yu H, Ma B, Huang Y, Ding Y, Liu Y, Ding Q, He C, Zhou J, Wang Y, Zhou G, Li Y, Yan Y, Hua J, Petersen B, Jiang Y, Sonstegard T, Huang X, Chen Y, Wang X. Base pair editing in goat: nonsense codon introgression into FGF5 results in longer hair. FEBS Journal, 2019, 286(23): 4675-4692

16. Yin K, Gao C, Qiu J L. Progress and prospects in plant genome editing. Nature Plants, 2017, 3(8): 17107 